\title{
Whats in a Name?
}

\author{
Sanjiv Nair
}

Received: 17 August 2011/Accepted: 18 August 2011/Published online: 31 August 2011

(C) Association of Oral and Maxillofacial Surgeons of India 2011

The Association of oral and maxillofacial surgeons of India (AOMSI) recently received suggestions regarding the proposal for a new postgraduate degree in maxillofacial surgery. The letter seeking approbation from the president on a new Master of Chirurgical (M.Ch.) qualification in oral and maxillofacial surgery seems to have been well drafted. But was it well thought out?

The Master of Dental Surgery (MDS) degree in oral and maxillofacial surgery (OMFS) is perhaps the most tenuous post graduate course in dentistry. By virtue of being a complex surgical field that frequently overlaps other surgical specialties in medical science we have had to fight at every step in our development. Unlike the other specialties of dentistry it has a significant interface with many super specialties like plastic surgery, otolaryngology, neurosurgery amongst others. For several years the AOMSI has desperately tried to restructure the curriculum and it certainly included the adding of another year to accommodate the extra needs. The proposals have been consistently shot down by the other branches of dentistry whenever it was brought up in the Dental Council of India (DCI).

We now have a MDS degree which trains dental undergraduates in the art and science of OMFS. This course has metamorphosed over the years from a 2-year course dealing primarily with dentoalveolar surgery to a 3-year programme incorporating advanced maxillofacial surgery. Today the specialty has to deal with the complexities of improving training and identify the deficiencies within the existing framework. We need to question whether starting a new post graduate course with a new name is the answer to our situation.

S. Nair $(\bowtie)$

Bangalore Institute of Dental Science, Bangalore, Karnataka

e-mail: snmaxfax@gmail.com
There are more questions than one can answer in a hurry. What would seem like the ideal module for training in OMFS? Should it incorporate basic medical training as part of its syllabus? Should it require dual degree as basis for advanced training? I think the best way forward is to learn from the training errors of other major centres/regions around the world.

Let us look at OMFS training in the United Kingdom (UK) and rest of Europe. The dual qualifications followed by five plus long years of higher surgical training before one is independently allowed to practice, are a luxury we can simply not afford in our country. The economic costs involved in undergraduate education and inadequate financial compensations received during training preclude such a notion.

The American OMFS training programs are expected to meet the same accreditation criteria. The programs can be either 4 or 6 years long. A 4 -year program offers a certificate in OMFS training, and the 6-year program integrates a medical degree within the residency. Regardless of the track, the training in OMFS remains the same. Some programs have residents in both tracks simultaneously.

Considering the above pathways as case studies let us debate on the merits and demerits of the two. The English training though exhaustive, is a system which we can simply not afford. The American system with its all in one residency sounds more practical but has more and more OMF surgeons settle into dentoalveolar surgery and implantology. This however has seen some change in the recent past with head and neck fellowship programmes being started in OMFS centres.

After evaluating all the training tracks, it does seem ideal to follow the American-way with or without an MD incorporated. The fears of our medical colleagues that this is a shortcut to earn a medical degree needs to be 
addressed. Hence a structured medical training not a medical degree should be included in the training pathway. To dwell upon the medical faculty to adequately train our postgraduates in general surgery, general medicine and other disciplines required for their foundation in maxillofacial training should be mandatory. This followed with fellowships in oral oncology and micro-vascular surgery, cleft and craniofacial surgery etc., would prepare the specialty for tomorrow.

In conclusion what we need is an MDS which has sufficient number of years to train in basic medical subjects not included in their Bachelor of Dental Surgery (BDS) curriculum along with higher surgical training in maxillofacial surgery. This can then be advanced with fellowships in desired sub-specialties like cleft and craniofacial or oncology. Not another degree with a fancy name.

Acknowledgment The author wishes to acknowledge the following academicians for their valuable input. Dr. George Paul, Salem, India; Dr. Deepak Gopalakrishnan, Cincinnatti, USA; and Dr. Satheesh Prabhu, Manchester, UK

Conflict of interest No conflict of interest whatsoever. 